Archives

\title{
D'un bon usage de l'équivoque : les avantures de Dassoucy
}

\section{Guy Catusse}

\section{(2) OpenEdition}

\section{Journals}

Édition électronique

URL : http://journals.openedition.org/ccrh/241

DOI : $10.4000 /$ ccrh.241

ISSN : 1760-7906

Éditeur

Centre de recherches historiques - EHESS

Édition imprimée

Date de publication : 1 avril 2004

ISSN : 0990-9141

Référence électronique

Guy Catusse, "D'un bon usage de l'équivoque : les avantures de Dassoucy », Les Cahiers du Centre de Recherches Historiques [En ligne], 33 | 2004, mis en ligne le 05 septembre 2008, consulté le 01 mai 2019. URL : http://journals.openedition.org/ccrh/241; DOI : 10.4000/ccrh.241

Ce document a été généré automatiquement le 1 mai 2019.

Article L.111-1 du Code de la propriété intellectuelle. 


\title{
D'un bon usage de l'équivoque : les avantures de Dassoucy
}

\author{
Guy Catusse
}

\section{NOTE DE L'AUTEUR}

Les participants au séminaire de Jean-Pierre Cavaillé « Virulence du libertinage » (E.H.E.S.S. 2002-2003), retrouveront ici l'écho des séances consacrées au « cas Dassoucy ».

Cecy paroistra obscur à ceux qui n'entendent pas

la musique.

Dassoucy, Les Pensées dans le Saint-Office de Rome,

Dassoucy entreprend le récit des "avantures " qui ont marqué ses années d'errance à travers la France et l'Italie alors qu'il est encore à Rome et songe à retourner à Paris ${ }^{1}$. Quelles sont ses intentions? Écrire un récit de voyage sur le mode burlesque qui lui est familier ${ }^{2}$ ? Sans doute : au moment où il s'apprête à regagner son pays après plus d'une dizaine d'années d'absence, il songe, selon toute vraisemblance, à se rappeler au bon souvenir des mécènes et des lecteurs qui ont jadis assuré un estimable succès à ses chansons et à ses romans. Cependant l'essentiel n'est pas là, mais dans la décision qu'il prend alors d'affronter la rumeur infamante attachée à son nom depuis que Loret dans sa Muse historique et Chapelle et Bachaumont dans leur Voyage ont révélé les accusations de sodomie qui l'ont conduit dans les cachots de la prison de Montpellier, en 1656. C'est probablement la publication à Cologne, en 1663, du Voyage de Chapelle et Bachaumont ${ }^{3}$ qui est à l'origine de la décision de Dassoucy de ne plus laisser sans réponse des accusations qu'il avait jusque là négligées. Je vois une confirmation de cette origine polémique des Avantures dans le soin qu'il a pris de dater très précisément la lettre de protestation qu'il adresse à Chapelle et qu'il nous dit lui envoyer dans le présent même de la narration: 
Lecteur humain, permets-moy de reposer et de prendre un peu d'haleine aux pieds de ce rocher, pendant que tu liras cette Lettre que j'envoy à mon amy Chapelle, avec la réponse à son libelle :

De Rome, le 25 juillet 1665

A Monsieur Chapelle. (Av., p. 175).

Quoi qu'il en soit de la réalité référentielle de cette lettre et de son envoi, retenons que Dassoucy a voulu confondre dans un même temps, la narration de ses infortunes de voyage et sa riposte aux attaques de Chapelle et consorts. Il convient donc de lire les Avantures comme un texte réactif destiné avant tout à récuser la sulfureuse réputation que lui ont faite ses anciens amis.

\section{Une audacieuse entreprise apologétique}

2 L'ouvrage est terminé au moment de son retour en France en 1669 et, dès 1670, il obtient le privilège qui lui permet d'en envisager une publication prochaine ${ }^{4}$. Le récit ne sera pourtant imprimé qu'en 1677, l'année de sa mort ${ }^{5}$. Dans l'intervalle, il l'a profondément remanié et récrit en fonction des nécessités et des aléas de sa vie chaotique. Mais, en dépit des transformations qu'il fait subir à son texte et d'une situation sociale enfin stabilisée (depuis 1674 il est pensionné au titre de «musicien du roi »), la visée reste la même : elle subordonne le projet poétique à l'intention apologétique :

Je n'escris point ces choses pour te faire admirer mon bel esprit : mais pour te faire admirer mes estranges Avantures et me faire connoistre un peu mieux à bien des gens dont je ne suis pas trop bien connu (Av. It., « Préface », p. 209).

confie-t-il à son lecteur, avant d'ajouter :

Je sçay bien que ce n'est pas une petite entreprise pour un particulier comme je suis de vouloir intéresser le public à lire son Histoire; c'est sans doute tout ce que le plus grand homme et le plus nécessaire à l'Estat pourroit faire [...] : aussi c'est par cette genereuse audace qui est fondée sur une suite de persecutions qui n'ont point d'exemple, que j'espere me rendre considerable à la postérité (Ibid,. p. 209-210).

L'entreprise, en effet, ne manque pas d'audace : non seulement Dassoucy transgresse les règles de la bienséance sociale qui réservent l'écriture autobiographique aux seuls individus dont la vie concerne la res publica, mais encore les « estranges avantures » qu'il se propose de publier sont celles qui l'ont conduit plusieurs fois en prison ${ }^{6}$ et ont associé à son nom l'image scandaleuse d'un sodomite doublé d'un impie. Or, même s'il sait que ses protestations de vertu et de piété ont peu de chance de convaincre des lecteurs auprès desquels il est, depuis longtemps, perdu de réputation, il refuse d'en démordre : il est la victime innocente que poursuivent de leur haine injustifiée «l'iniquité des méchans, l'ignorance des simples et la malice des sots » (Av., p. 28). On ne le croit pas? Tant pis. L'essentiel, pour Dassoucy, n'est pas dans un combat incertain pour faire reconnaître son improbable innocence; le véritable enjeu de son récit, c'est la lutte qu'il mène, avec la plus farouche énergie, contre l'ensevelissement de l'homme et du poète - c'est tout un - sous la réputation infamante du débauché. En revenant, à sa manière, sur ses " estranges avantures", il vise moins à se disculper qu'à s'affirmer aux yeux de ses contemporains et de la " postérité » et, pour cela, il compte davantage sur les séductions de son talent de plume que sur la force persuasive de ses douteuses dénégations.

Emprisonné plusieurs fois pour ses mœurs et/ou son irréligion, trahi par "l'amy Chapelle », déshonoré par la rumeur publique, exclu de la liste des écrivains estimables par Boileau, il refuse de se laisser déposséder de sa dignité d'homme et de poète. On a 
voulu l'anéantir? Il va s'exhiber dans une équivoque mise en scène de soi à travers laquelle, dans un même geste ostentatoire, il s'affiche et se masque tout à la fois.

Chapelle avait «malicieusement » ironisé sur le jeune chanteur qui l'accompagnait au moment de l'affaire de Montpellier et ses vers assassins ${ }^{7}$ avaient été repris et amplifiés par « cette vermine picquante toûjours obsedée de l'impertinence du Demon de l'oüyrdire » (Av., p. 31). Au lieu de s'en tenir à une catégorique protestation d'innocence ou à un discret silence, Dassoucy se risque, dès les premières lignes de son récit, à l'équivoque provocatrice :

Quoy que je ne sois ny Comte ny Marquis, je ne laissois pas d'avoir deux Pages à ma suite [...]. Ces Pages estoient de ceux qu'on appelle Pages de musique, autrement des chantres à chausses retroussées ( $A v$., p. 34).

6 Quelques chapitres encore et il fera dire à Pierrotin, un des deux pages «à chausses retroussées » qui se plaint des mauvais traitements de son maitre: «il me traite à la Turque » (Av., p. 86) ce que tout lecteur averti des sens que peut prendre l'expression dans d'autres contextes, ne manquera pas de traduire : il me traite en sodomite.

7 Telle est la stratégie de défense que Dassoucy a choisie: ne rien céder à l'accusation, coiffer le bonnet du fou et jouer de toute la gamme de l'équivoque pour s'autoriser les plus insolentes provocations. Mais sous ce travestissement de bouffon son objectif essentiel est bien de désamorcer la calomnie et de revendiquer son droit à la considération sociale, sinon pour ses mœurs tout au moins pour son talent de plume :

$\mathrm{Au}$ reste, ne t'attends pas que je m'aille insinuer dans ton esprit par de belles parolles, pour t'obliger à pardonner à mes deffauts, ny pour te faire remarquer dans les choses les plus basses la qualité de mes expressions, qui possible ramperoient dans une autre plume que la mienne. Je sçay que tu me feras justice (Av., «Préface ", p. 32).

La formulation ambivalente de la dernière phrase appelle le lecteur à reconnaitre tout à la fois l'excellence de l'écrivain et la respectabilité de l'homme.

\section{Équivoques burlesques}

8 Dans la mesure où elle concentre l'intérêt sur la virtuosité de l'énonciation plus que sur la vérité de l'énoncé, l'écriture burlesque est parfaitement adaptée à cette stratégie de défense qui se soucie davantage d'esquiver les questions que de réfuter les accusations. Une scène rejoue, dans le texte, cette stratégie du langage équivoque utilisé pour déjouer la suspicion. Il s'agit de l'interrogatoire de type inquisitorial auquel l'hypocrite Triboulet soumet Pierrotin pour lui faire avouer que son maitre est un débauché :

[...] je vous demande si c'est un homme de vertu ou un homme débauché et de mauvaise vie. - Non, dit Pierrotin, je ne connois point d'homme de meilleure vie que luy, surtout quand il a de l'argent. (Av., p. 87).

9 Au-delà du personnage de Triboulet, la réplique rouée de Pierrotin s'adresse à la personne du lecteur-inquisiteur tenté d'en savoir davantage sur les mœurs ou les convictions religieuses de Dassoucy. Il ne recevra pour toute réponse à ses insidieuses questions que des boutades burlesques et des paroles amphibologiques. S'interroge-t-il, par exemple, ce lecteur malveillant, sur les soupçons de sodomie qui pèsent sur Dassoucy? Il n'obtiendra ni une franche dénégation ni, bien sûr, un aveu impensable dans une société où la sodomie est un crime passible du bûcher. Il devra se contenter de prendre connaissance de l'équivoque protestation que le narrateur-héros des Avantures 
adresse, comme un pied de nez, aux « Précieuses de Montpellier » qui l'accusent de leur préférer les garçons :

J'adore toute la nature [...]

Me pouvez-vous haïr sans cause

Moy qui ne vous fis jamais rien? (Av., p. 142).

Et si la pirouette ne lui suffit pas, le lecteur suspicieux se verra opposer cette autre preuve d'orthodoxie, bien dans la tradition grivoise des jeux de mots burlesques :

[...] les femmes galantes [...] m'appelloient heretique non en fait de religion mais en fait d'amour [...] sans se ressouvenir de tant de serenades que je leur avois données et de tant de tendresses que j'avois eues pour elles, quand dés mes plus jeunes ans, passant à Montpellier, je leur enseignois à joüer du Luth, et leur mettois la main sur le manche... (Av., p. 139-140).

À curiosité déplacée, réponse bouffonne. Les rieurs sont de son côté, l'objectif est atteint : Dassoucy a rendu le lecteur complice de sa duplicité. Sans doute ne l'a-t-il pas convaincu de l'orthodoxie de ses mœurs, mais était-ce bien son objectif? S'il visait vraiment à se disculper des accusations d'homosexualité, comment comprendre, quelques pages plus loin, cet aveu, à peine voilé sous l'ambiguïté de la formulation, des liens d'intimité qui le liaient jadis au jeune Chapelle :

[...] il me cedoit fort librement la moitié de son lit. C'est pourquoy, aprés avoir eu de si longue preuve de la qualité de mes desirs et m'avoir bien daigné honnorer plusieurs fois de sa couche, il me semble que c'estoit plutôt à luy à me justifier qu'à Messieurs du Presidial de Montpellier, avec lesquels je n'ay jamais couché (Av., p. 198-199).

Comprenne qui voudra. Pour ce qui concerne ses mœurs, Dassoucy n'en dira pas davantage. Il ne faut attendre de lui ni mea culpa repentant, ni réfutation crédible. Passé maitre dans l'art de la diversion, un même usage de la formule équivoque lui sert tout à la fois de voile et de dévoilement ${ }^{8}$.

11 En revanche, quand il s'agit de protester contre les accusations d'impiété dont on le charge, il semble prendre le contre-pied de cette posture de défense équivoque fondée sur l'esquive, et choisit une attitude résolument offensive: pas une "avanture", pas une préface, pas une épître dédicatoire, qui ne lui soit une occasion de rappeler sa totale orthodoxie religieuse. L'équivoque naît ici de l'outrance. Derrière le masque outrageusement peinturluré d'un fidéisme à toute épreuve, on n'a pas un grand effort à faire pour deviner le visage du libertin.

Dès la « Préface » des Avantures, Dassoucy affiche ses intentions édifiantes :

[...] ne dédaigne point de m'accompagner jusqu'à la fin de mon voyage, dont la suite miraculeuse te fera un tableau de la vie humaine d'autant plus digne d'estre conservé, que c'est dans ce tableau sans exemple que les enfans de tes enfans y apprendront en se divertissant, non seulement la science du monde, mais la science du Ciel qui est la science des sciences (Av., p. 32).

Le lecteur le plus complaisant aura quelque peine à prendre au sérieux cette promesse de révélation des secrets du Ciel. Le récit est ainsi tout entier saturé de références à Dieu, aux anges, à l'Évangile, au salut de l'âme, aux révélations, aux miracles de la Providence ou à la malignité du Démon. L'ironie perce derrière l'outrance de ces déclarations hyperboliquement orthodoxes. Trop, c'est trop. L'excès appelle son retournement critique.

On trouvera un exemple de cette emphase suspecte dans l'épisode où le narrateur-hérosDassoucy, qui voyage en chrétien avisé, un livre d'heures dans sa poche, une « petite croix d'or » suspendue au cou, est agressé par un groupe de bigotes qui l'accusent d'être, tout à 
la fois, un "Juif », un " réprouvé », un " parpaillot » et un " ateistre » (sic). Pour se tirer d'un procès aussi mal engagé, il exhibe à ses calomniatrices médusées et à tous ceux qui assistent à la scène, les preuves de sa parfaite foi catholique :

[...] estant écolier estudiant sous les Jésuites, j'avois toujours emporté le prix de doctrine Chrestienne je possedois encore si bien mon Catechisme et le sçavois encore si bien par cœur que je l'aurois dit à rebours. C'est pourquoy, les voyant disposez à m'écouter, je leur en recitay, pour le moins trente pages sans hésiter aucunement ny manquer une syllabe (Av., p. 88).

Et on voudrait douter de la sincérité de sa foi ? Le voilà en tout cas protégé des censeurs qui, quoi qu'ils en pensent, se trouvent désarmés devant l'affirmation de cette irréprochable orthodoxie. Pour le reste, si le soupçon pouvait naître, dans des têtes malveillantes, qu'on est là en présence d'un cas avéré d'ironie irrévérencieuse, Dassoucy pourra toujours s'abriter derrière les droits du burlesque à l'outrance comique : ce que dit un bouffon ne tire pas à conséquence.

On retrouve une même utilisation retorse du caractère discordant de l'écriture burlesque dans l'évocation des nombreuses occasions qui lui ont été données de bénéficier de l'intervention divine. Autour d'une table de jeu, par exemple, où Dieu vient fort opportunément à son secours dans ce qu'il faut bien appeler une supercherie : il a perdu jusqu'à son dernier «teston » et devrait donc quitter le jeu comme le fait tout honnête homme qui n'a plus les moyens de miser; au lieu de cela, il trompe ses partenaires et continue la partie en leur laissant croire qu'il a encore une grosse somme d'argent en poche. Lisons la suite, elle est édifiante :

[...] à moins d'un petit miracle, je ne voyois point comment je pourrois sortir a mon honneur d'une si douteuse et si mauvaise affaire : aussi la Providence, qui se sert de tout, et qui est toûjours au guet pour maintenir ses creatures, ne manqua point à faire ce petit miracle à point nommé ; [...] au bout d'une petite demy-heure je me vis en possession de quinze petites pistolles, mais bien de poids, que je contemplois avec autant d'étonnement que si elles fussent tombées des nuës, comme en effet elles venoient du Ciel. Aussi comme il ne faut pas abuser des grâces que Dieu nous envoye [...] je me retirai avec ce petit gain... (Av., p. 167-168).

Et comme cette irréfutable marque d'élection ne suffisait sans doute pas, Dieu, qui lui a fait la grâce d'un miracle en le tirant d'une situation déshonorante, va encore l'encourager à persévérer dans sa coupable passion du jeu. C'est ainsi que les jours suivants, « le Ciel, qui dispose de la fortune » (ibid) lui permet de refaire rapidement la sienne! Voilà des propos dont l'impertinence bouffonne ne manque pas d'audace dans un temps où sévit une sourcilleuse et redoutable censure religieuse.

Plus risqué encore est ce rapprochement des miracles du Christ avec des actes de sorcellerie et de magie :

[...] croyez-vous que Dieu qui fit les sots et les sages et qui est venu mourir autant pour les uns que pour les autres eust jamais fait tant de merveilles sans les sots, qui ne voulant pas croire à ses paroles, l'appeloient Sorcier et Magicien [...] et n'estoitce pas assez d'avoir ressuscité le Lazare, pour faire connoistre à tous ce qui estoit bien sensé, qu'il estoit un Dieu ? (Av., p. 121-122).

Jésus répondant au défi des "sots", obligé de multiplier les miracles pour prouver sa nature divine, le nom de «Dieu » rapproché, dans une même phrase, de celui de «Sorcier» ou de "Magicien", même s'il s'agit par là de dénoncer la malignité de «Messieurs les sots» (dont Dassoucy se dit lui-même-comme Jésus!-la victime innocente), on n'en est pas moins ici aux marges de la parole blasphématoire. 
On pourrait multiplier les exemples où, à peine dissimulée derrière la discordance burlesque, s'affiche une approche dérisoire et libertine des miracles et des mystères divins9. On ne trouvera pas, dans tout l'ouvrage, une seule référence à Dieu ou à la religion qui puisse laisser penser que Dassoucy est sincère quand il proclame sa foi. Les situations au cours desquelles il affiche une attitude dévote sont autant $d^{\prime}$ ' avantures » où la dignité du sujet abordé est subvertie par la dérision de la forme.

On est d'ailleurs d'autant plus en droit de douter de la sincérité de sa foi, qu'il nous donne lui-même une indication sur la façon dont il en use avec la religion. Il est à Turin et cherche toutes les occasion de faire apprécier ses talents de poète-musicien par Madame Royale dont dépend son " établissement » à la cour de Savoie ${ }^{10}$ :

[...] ayant maintefois oüy dire qu'on n'entre point en Paradis malgré les Saints, je voulus, pour me les rendre propices, faire encore Musique en sa chapelle. Soit qu'elle ouïst la Messe dans sa chambre, au Saint-Suaire ou en quelque autre Eglise, je la suivois partout comme un barbet [...]; par ce moyen je devins en peu de temps la plus dévote personne du monde; car il ne faut pas croire que cette pieuse Princesse qui pleuroit ordinairement aux Autels, eust crû satisfaire aucunement sa piété, assistant à une seule messe ; il lui en faloit tous les jours pour le moins deux et le plus souvent trois, durant lesquelles je faisois trés-longue et trés-dévote Musique, toûjours à deux genoux. Juge, Lecteur, si je ne devois pas estre tout à Dieu; cependant je t'assure que la chose à quoy je pensais le moins c'estoit de l'importuner de mes prieres [...]; et, quoy que les paroles que je murmurois entre mes dents fussent toutes saintes et sacrées, ce n'étoit point tant pour la gloire de Dieu que je les voulois unir à mes chants que pour la satisfaction de cette Divinité mortelle: qu'alors moi, malheureux, j'eusse préféré à la Divinité mesme (Av. It., p. 252).

Je propose de lire ce désinvolte aveu d'opportunisme religieux comme un indice de la confession libertine de Dassoucy. Sous le travestissement de l'histrion avant tout préoccupé de plaire à ses maîtres, il invite son lecteur à conclure qu'il lui en coûte peu de murmurer, "toujours à deux genoux", des paroles «saintes et sacrées", s'il peut en attendre un profit.

Ici, une question mérite d'être posée : Dassoucy ne se révèle-t-il pas, dans cette anecdote, semblable à ces faux-dévots qu'il dénonce par ailleurs si violemment et dont il nous présente le portrait détestable sous les traits de Triboulet ? ${ }^{11}$ Ne serait-il pas lui-même une figure représentative de ces " véritables athées » que sont, selon ses propres paroles, les hypocrites ? ${ }^{12}$

Si l'on considère avec lui que l'hypocrite est un homme :

[...] qui n'a dans la bouche que des feux, des potences et des roües et qui sonne partout le tocsin sur le pauvre pecheur (Av., p. 93).

on peut affirmer que Dassoucy ne fait pas partie, à l'évidence, de ces Tartuffe-là.

Mais s'il s'agit de voir dans l'hypocrite un de :

[...] ces mocques-Dieu qui sont les veritables ennemis de sa puissance et de sa gloire, et qui de son Estre s'en font une chimere dont ils se targuent en public et se joüent en secret (ibid).

alors l'hypothèse de la duplicité hypocrite de ses proclamations dévotes ne peut être repoussée sans examen : les Avantures n'affichent-elles pas publiquement une foi naïve et sans faille que ce que l'on connaît de la vie et de la personnalité de Dassoucy rend assez peu vraisemblable ${ }^{13}$ ?

21 C'est, me semble-t-il, dans la mesure où l'outrance même de ses déclarations discrédite ouvertement ses protestations fidéistes que Dassoucy peut être disculpé des accusations 
d'hypocrisie. D'ailleurs, à d'autres moments du récit, loin de toute attitude hypocrite, il donne des indices de son scepticisme religieux. C'est par exemple le cas dans l'importante «Épître aux sots » qui ouvre le deuxième volet des Avantures où, au détour d'une phrase dans laquelle il fustige ceux qui ont pris pour argent comptant les accusations de Chapelle, il glisse, sur un ton qui n'a rien de burlesque ni d'équivoque :

Dieu, quelle pitié ! nous avons tant de peine à croire à l'Evangile, et nous n'avons point de honte de donner nostre creance aux plus méchants de tous les hommes! ( Av., p. 124) $)^{14}$

"Nous avons tant de peine à croire à l'Évangile » : on est bien loin de cette " croyance du Charbonnier $»^{15}$ dont il prétend faire sa profession de foi. Faut-il conclure à l'inconséquence, voire à la palinodie d'un bouffon qui ne recule devant aucune provocation verbale pourvu qu'elle prête à une douteuse pitrerie, mais qui se rétracte au premier froncement de sourcil de ses maîtres? Un jugement aussi méprisant, dans la ligne de toute une tradition de l'historiographie du libertinage, se trouve sous la plume d'un des pionniers du genre, Victor Fournel qui ne s'embarrasse pas de nuances pour trancher : «Lisez les Pensées du Saint-Office si vous voulez savoir jusqu'à quelle profondeur de mysticisme et de mélancolie peut descendre l'âme d'un bouffon qui a peur. $»^{16}$

Ont-ils bien lu Dassoucy, ces contempteurs érudits ${ }^{17}$ ? Écoutons-le définir son burlesque et nous en donner les clés de lecture :

[...] ce n'est pas encore assez qu'il soit fin dans ses pensées et plaisant dans ses rencontres, il faut que, sur peine de servir de bouffon aux Laquais et de divertissement aux Servantes [...] il soit concis, figuré et encore mistique, s'il est possible, comme on peut voir dans tous mes ouvrages burlesques, où le sens qui est caché vaut souvent mieux que le sens litteral. [...] aussi, ce n'est pas pour tout le monde que j'escris ainsi, mais seulement pour ceux qui ont assez de finesse pour me dechiffrer (Av. It., p. 276-277).

Il n'est pas seulement ici question de cette "substantifique moelle " que Rabelais nous invite à chercher sous l'enveloppe "grotesque » de la forme, mais de véritable «sens caché ", « mistique ${ }^{18}$, réservé à ceux qui savent lire sous les lignes. Dans la dédicace à Marguerite d'Orléans qui ouvre la deuxième édition, expurgée, des Rimes Redoublées ${ }^{19}$, Dassoucy dénonce les rigueurs de la censure avec lesquelles il se voit contraint de composer :

Grace à la modestie du temps et à la sainteté du Siècle, ce livre est tellement épuré qu'il ne craint plus la coupelle $^{20}$.

C'est dans des termes très proches que, dans les Avantures, il fait connaître à son lecteur les raisons pour lesquelles il renonce à publier la «relation de la tragi-comique avanture » de Montpellier, qu'il avait écrite à sa sortie de prison,

[...] et que je t'aurois donnée dans son lustre, si le temps devenu plus chagrin ou pour mieux dire plus modeste m'eust permis de te faire rire sur un sujet de cette nature (Av., p. 152-153).

Il doit donc bien être entendu que Dassoucy écrit sous contrainte et qu'il est en droit d'attendre de son lecteur qu'il le considère avec moins de condescendance que Victor Fournel qui lui trouve "l'âme d'un bouffon» ou René Pintard qui l'affuble du titre dérisoire de "poétereau " et juge que "sa métaphysique se borne à une médiation assidue sur le goût de l'épaule de mouton. ${ }^{21}$ Misère d'un texte burlesque lu sans «finesse» où, de la tonalité bouffonne de l'œuvre et de l'autoportrait qu'elle nous présente du narrateur-héros, on conclut à la «burlesquerie $»^{22}$ de celui qui l'a écrite. Après quoi, il ne reste plus qu'à faire retour sur le récit pour décréter qu'un individu aussi 
dérisoire n'a pu écrire autre chose qu'une œuvre médiocre, sans originalité et sans profondeur ${ }^{23}$.

\section{Équivoques fictionnelles}

Cette confusion entre la personne et le personnage tient à l'équivoque autobiographique entretenue par Dassoucy lui-même qui ouvre la «Préface» des Avantures sur cette déclaration ambivalente :

Je suis le Héros veritable de mon Roman, qui aprés avoir long-temps vogué contre vent et marée sur une mer orageuse, ay finallement attrappé un heureux port (Av., p. 31).

Dans le temps même où il est énoncé, le "pacte autobiographique ${ }^{24}$, garant de la référentialité du récit, sinon de sa vérité, est démenti par le pacte de lecture fictionnel qui s'y superpose. Il n'est plus alors question que de «héros » et de «roman» dans un contexte métaphorique dont le référent n'est pas de l'ordre de la réalité mais de la fable odysséenne.

Le trouble du lecteur vient de cette difficulté à distinguer la part fictionnelle de la part référentielle du récit. Or, il s'agit d'un enjeu de lecture essentiel. On ne peut lire les Avantures comme on lit ces autres romans écrits à la première personne que sont, par exemple, Francion, le Page disgracié ou Les Etats et Empires de la lune. C'est pourtant ce que font de nombreux commentateurs. Dans sa volonté de classer Les Avantures dans ce qu'il appelle le «roman canaille », Jacques Berchtold pousse la confusion jusqu'à écrire : « Et même lors de son voyage en Italie, d'Assoucy subira encore - comme Francion - une incarcération romaine, cette fois dans les geôles du Saint-Office. $»^{25}$. C'est vouloir ignorer que Francion est un « être de papier » et Dassoucy un être réel qui a souffert dans sa chair d'un emprisonnement de quinze longs mois dans le "pieux enfer $»^{26}$ des cachots de l'Inquisition. Sorel, Tristan et Cyrano, utilisent des éléments autobiographiques pour nourrir leur fiction (ce que font, à des degrés divers, tous les romanciers) ; Dassoucy, lui, a recours à des procédés fictionnels pour mener à bien son « entreprise » autoréférentielle ${ }^{27}$. Sans doute son jeu avec les codes fictionnels participe-t-il de l'opération de séduction poétique sans laquelle il serait un « écrivant » et non un " écrivain ${ }^{28}$, mais cette fonction de divertissement poétique est ici mise au service d'un plaidoyer pro domo.

$\mathrm{Si}$, avec Philippe Lejeune, on fait de l'«identité de nom » entre l'auteur, le narrateur et le héros, la marque d'une séparation radicale entre l'autobiographie et la fiction ${ }^{29}$, alors, seul parmi les auteurs que nous venons d'évoquer, Dassoucy répond à ce critère discriminatoire. Son nom de plume est constamment rappelé dans le titre en forme de résumé qui introduit chacun des chapitres de son récit ${ }^{30}$; à divers moment du voyage, plusieurs personnages de rencontre, reconnaissant notre narrateur-héros, ne manquent pas de l'identifier comme étant le poète Dassoucy que ses romans burlesques et ses chansons ont rendu célèbre ${ }^{31}$; enfin, l'auteur-narrateur prend soin de rattacher explicitement son pseudonyme à son véritable patronyme et déclare, contre toute vérité :

Il est vray que mon père s'appelloit Coypeau, mais il s'appelloit encore Dassoucy, et

je m'appelle Dassoucy Coypeau comme mon père (Av., p. 158).

Quelques pages auparavant, alors qu'il évoquait un épisode douloureux et humiliant de son enfance, l'affirmation orgueilleuse de son nom-patronyme et pseudonyme confondus - lui servait à revendiquer sa dignité face au mépris aristocratique de «Don Diego », le fils tyrannique du noble espagnol qui l'avait pris à son service : 
[...] moy donc, Charles Coypeau Dassoucy, je n'estois pas moins Don diable que Don

Diego (Av., p. 130).

Les choses seraient donc simples et univoques si cette revendication insistante du nom n'était elle-même prise dans la trame d'un récit que l'auteur a qualifié de "roman » et dont le caractère fictionnel subsume chacun des éléments qui le composent. La séquence «Charles Coypeau Dassoucy » renvoie bien à une réalité référentielle extérieure au texte mais rien ne nous assure que ce qui nous est dit de cette réalité relève de la vérité : le père de Dassoucy, par exemple, s'appelait bien Coypeau mais il ne s'est jamais appelé Dassoucy.

Un semblable brouillage entre réalité et fiction se retrouve dans le jeu du péritexte où il convient de distinguer le «vrai » Dassoucy, celui qui dédie son ouvrage au "vrai» Louis XIV :

Sire,

J'aurais été un Poëte bien fol et ma Muse bien insensée si, après l'honnheur qu'elle a

receu de divertir tant de fois Votre majesté... (Av., p. 25).

et le Dassoucy de la «Préface, Au Lecteur » dont le «je » n'a d'existence que dans sa relation au «tu» du lecteur de fiction que l'écrivain Dassoucy fabrique, à la mesure de l'oreille complaisante dont il a besoin pour créer l'illusion de la confidence :

Aussi ce n'est pas pour eux [les «méchans »] que j'écris, mais pour toy, genereux

Lecteur, qui, justement indigné contre cette vermine picquante toûjours obsedée de

l'impertinence du Demon de l'ottyr dire... (Av., « Préface. Au Lecteur », p. 31).

Celui qui, dans cette même préface, proclame "Je suis le Heros véritable de mon Roman » est donc déjà entré dans le jeu de la fiction et nous n'avons pas plus de raison de prendre au pied de la lettre cette déclaration que celle qui lui succède, quelques pages plus loin, quand, déplaçant facétieusement les termes, il décide que c'est «Pierrotin, le Heros de mon véritable roman" (Av., p. 85). À quelle vérité nous renvoie ce qualificatif de "véritable ", sinon à la "feintise ludique " $^{32}$ du mode fictionnel ? Effets de miroir et de miroitement, jeux d'anamorphoses entre Dassoucy-écrivain et Dassoucy-héros, lecteur réel et lecteur fictif, texte et péritexte, rien n'est tout à fait l'image exacte de la réalité mais rien d'essentiel ne relève non plus de la pure imagination ${ }^{33}$.

31 Ce statut générique incertain redouble les effets d'indécision de l'équivoque burlesque. «L'autobiographie se définit autant par sa lecture que par son écriture » écrit Philippe Lejeune $^{34}$. Nous avons vu qu'on pouvait en dire autant du burlesque. Dans les deux cas, le sens est affaire de réception. Dassoucy en est parfaitement conscient qui fait dire à son narrateur-héros, en préambule à son récit :

Ly donc, et lisant profite de mes disgraces; ry, sage lecteur, et, tout riant de mes

folies, fay-toi encore plus sage à mes dépens. (Av., «Préface, Au Lecteur », p. 32).

C'est un souhait, presque une supplique : que le lecteur refuse de croire à la vérité de ses « disgraces » ou bien qu'il reste insensible à la «finesse » de son écriture burlesque et voilà Dassoucy ravalé au rang de " poètereau », et de "bouffon ». À chaque lecteur donc, le Dassoucy que sa lecture mérite. Et tant pis pour «ceux qui n'entendent pas la musique ».

C'est à cette souveraine responsabilité du lecteur que renvoie l'auteur de l'épître « $\mathrm{Au}$ lecteur en faveur de mon amy Chapelle » quand, s'agissant de savoir qui dit la vérité de Chapelle ou de lui-même dans le récit contradictoire que l'un et l'autre donne de l'affaire de Montpellier, il écrit, avec l'apparente désinvolture qui lui est habituelle :

Dassoucy nous rappelle opportunément que tout récit est une «fable » et que le lecteur reste maître du sens qu'il entend donner à ce qu'il lit. Soit. Mais dans le cas présent, au- 
delà du récit qui nous en est « conté », l'« avanture » de Montpellier renvoie à une réalité référentielle qui relève d'un jugement de vérité. Dassoucy (Charles Coypeau, le " vrai»), soupçonné de sodomie, a bien connu la prison de Montpellier et les accusations infamantes qui ont été portées contre lui ont bien été divulguées par Chapelle, Loret et quelques autres" ${ }^{35}$. Si fiction il y a, ici s'arrête la feintise et pas plus qu'on ne peut prendre au pied de la lettre ce que le narrateur Dassoucy nous raconte, on ne peut ignorer la réalité des " persécutions » dont l'auteur Dassoucy a été victime et qu'il a décidé de rendre publiques sous le couvert du double-(je)u auquel l'autorisent les codes de la fiction et de l'écriture burlesque.

Le recours à cette double équivoque, burlesque et fictionnelle, présente plusieurs avantages : il autorise un récit autobiographique que la condition sociale de son auteur ne suffirait pas à légitimer; il sert à enrober dans une rhétorique retorse un plaidoyer pro domo plus ou moins contraint ; il permet enfin - il permet surtout - à Dassoucy d'affirmer un droit à la dignité et à la reconnaissance sociale que ses accusateurs lui refusent. Là se situe, me semble-t-il, l'enjeu essentiel de cette " autobiographie aménagée $»^{36}$ que sont les Avantures, leur véritable visée apologétique. On peut lire la proclamation liminaire : «Je suis le Héros véritable de mon Roman ", comme l'affirmation de la volonté orgueilleuse de Dassoucy de se ressaisir tout entier, homme et poète, à travers l'écriture ennoblissante d'un récit parodique qui élève au rang d'«estranges avantures» une succession de déboires plus ou moins douloureux mais jamais aussi « estranges" qu'il veut bien les présenter. En ce sens, les Avantures relèvent d'une «entreprise " autobiographique de construction de soi. C'est à son art d'écrire que Dassoucy confie le soin d'affirmer sa respectabilité et c'est pour cela que sa défense du burlesque contre le jugement méprisant de Boileau ${ }^{37}$ et contre le goût du temps "devenu plus chagrin", revêt un caractère existentiel $^{38}$ dont l'enjeu excède la simple querelle littéraire ou la banale protestation d'une vanité d'auteur blessé dans son amour-propre.

On trouve dans les Avantures cette " énergie de parole » dans laquelle Roland Barthes ${ }^{39}$ reconnaissait la force de l'œuvre "baroque » et qui donne aussi toute sa vigueur à l'écriture burlesque ${ }^{40}$. C'est cette énergique affirmation de soi que, pour ma part, je retiens de la lecture des Avantures de Dassoucy. Au-delà de ses mœurs hétérodoxes et de ses opinions religieuses hasardeuses, c'est dans cette "généreuse audace » que je lis le libertinage d'un écrivain qui, dans un geste de défi, use de l'équivoque pour transgresser les règles de la convenance sociale et afficher, dans un même mouvement, sa singularité et sa liberté de pensée.

\section{NOTES}

1. Une première version des Avantures est déjà écrite au moment de sa condamnation par le tribunal du Saint-Office en mars 1667 puisqu'il attribue à la circulation imprudente de ce texte la cause de son arrestation : «J'estois sur le point de retourner en France et sans me souvenir que je n'estois point à Paris mais que j'estois encore à Rome, moy qui jusqu'à ce temps m'estois tenu 
clos et couvert, je fus assez simple pour faire voir mes adventures d'Italie à plusieurs personnes de qualité. » Charles Dassoucy, Rimes redoublées, Paris, Nego, 1671, p. 67.

2. C'est ce que pense Henri Coulet pour qui les Avantures et Avantures d'Italie sont «à ranger dans le genre bâtard et assez artificiel du voyage où s'unissent la vérité et la convention », Henri Coulet, Le Roman jusqu'à la Révolution, Paris, Armand Colin, 1967, p. 190.

3. Après avoir longtemps circulé sous forme de manuscrit, ce récit a été publié pour la première fois, sans autorisation, dans Recueil de quelques pièces nouvelles et galantes tant en prose qu'en vers..., Cologne, Marteau, 1663.

4. Sur la biographie de Dassoucy, voir Charles Eugène Scruggs, Charles Dassoucy: Adventures in the Age of Louis XIV, Boston, University Press of America, 1984.

5. Les Avantures de Monsieur Dassoucy, Paris, C. Audinet, 1677 et Les Avantures d'Italie de Monsieur Dassoucy, Paris, A. de Raffé, 1677. Je me réfèrerai ici à l'édition de ces deux œuvres par Émile Colombey dans Les Aventures burlesques de Dassoucy, Paris, Garnier, 1876 (première édition : Paris, Delahays, 1856). Jacques Prévost a donné une édition récente du premier de ces deux ouvrages, Les Aventures de Monsieur Dassoucy, dans Jacques Prévost, éd., Libertins du XVII siècle, Paris, Gallimard, "La Pléiade », 1998. Pour éviter d'alourdir les notes, j'utiliserai les abréviations suivantes: Av. pour Les Avantures et Av. It. pour Les Avantures d'Italie.

6. Après la prison de Montpellier en 1656, et celle du Saint-Office à Rome en 1667, il connaîtra les cachots du Châtelet où, accusé une fois encore de sodomie, il est emprisonné 6 mois en 1673. Il a alors 70 ans.

7. «[...] nous le questionnâmes donc malicieusement: «Ce petit garçon qui vous suit / et qui derrière vous se glisse / Que sait-il ? En quel exercice / En quel art l'avez-vous instruit? » / « Il sait tout dit-il. S'il vous duit, / Il est bien à votre service. » Voyage de Chapelle et Bachaumont, in Euvres de Chapelle et de Bachaumont, nouvelle édition par Tenant de Latour, Paris, Jannet, 1854, p. 97-98.

8. René Démoris voit dans la révélation à demi voilée de ses rapports homosexuels avec Pierrotin « une sorte d'involontaire défi, contraire au projet avoué » (René Démoris, Le Roman à la première personne, Genève, Droz, 2002 p. 127). Je ne partage pas ce dernier point de vue. Je plaide au contraire pour la totale lucidité de Dassoucy, maitre de sa parole et conscient de ce qu'il dit comme de ce qu'il tait. À l'interlocuteur-confident qui, dans La Prison de Monsieur Dassoucy, lui reproche de se laisser aller à la raillerie sans discernement, il répond avec vivacité : «J'en prens à témoin le Seigneur, / Je sçais parler et sçais me taire». (Les Aventures burlesques..., op. cit., p. 422) Je propose de prendre cette protestation au sérieux.

9. Un dernier exemple, parmi bien d'autres : dans la nuit la plus obscure, perdu dans une forêt, notre héros cherche désespérément la bourse qu'il a égarée en voulant la dissimuler aux yeux de cavaliers qu'il a pris pour des voleurs. Dans ce contexte burlesque, un mystérieux bûcheron apparaît soudain, venu d'on ne sait où, une lanterne à la main, le conduit quelques mètres plus loin et, lui désignant un endroit précis, il lui déclare, avec cette simplicité biblique qui est la marque des miracles christiques : «Viens, mon amy, rend graces à Dieu et prends ton argent. » Et Dassoucy récupère sa bourse! (Av., p. 82).

10. Voir le titre en forme de résumé du chapitre X des Avantures d'Italie: «Dassoucy, pour obtenir, auprès de leurs Altesses Royalles, l'establissement qu'on lui avoit fait espérer, fait une plaisante description de son affection et de son assiduité. »

11. C'est le point de vue - opposé à celui que je défends ici - de Madeleine Alcover : « [...] masqué comme un tartuffe lorsque son intérêt l'exige, le héros reçoit comme un boomerang la condamnation, par le narrateur, du faux-dévôt Triboulet. Cette faille fait se craqueler tout l'édifice de la défense : le lecteur ne peut pas, à moins de se crever les yeux, se fier à un narrateur qui lui présente, d'une manière comique de surcroît, un héros qui souffle le chaud et le froid selon les circonstances ». Comme je ne considère pas, contrairement à Madeleine Alcover, que «l'édifice de défense » de Dassoucy repose sur l'espoir que le lecteur puisse jamais croire à la 
sincérité de ses vertueuses protestations d'innocence, je me refuse à voir dans Les Avantures, une apologie inconséquente, minée par les contradictions du narrateur. Cette façon de considérer Dassoucy comme un piètre avocat de sa propre cause me paraît discutable. Madeleine Alcover, « Un gay trio : Cyrano, Chapelle, Dassoucy », in R. Heyndels et B. Woshinsky (éd.), L'Autre au XVII siècle. Actes du $4^{\mathrm{e}}$ colloque du Centre international de Rencontres sur le XVII ${ }^{\mathrm{e}}$ siècle (University of Miami, 23 au 25 avril 1998), Biblio 17, vol. 117, Tubingen, 1999, p. 265-275.

12. «[...] ce sont là les veritables athées que nous cherchons en vain parmy les plus débauchez et que nous ne trouvons pas mesme parmy les plus scelerats de tous les hommes, puisque, hors de l'hypocrite, je ne croy pas qu'il y ait aucun homme sur la terre qui ne reconnoisse la Divinité et qui n'encense ses Autels. » (Av., p. 93).

13. Cette apparente duplicité semble culminer dans Les Pensées de Monsieur Dassoucy dans le Saintoffice de Rome qu'il publie séparément en 1676 mais qu'il avait d'abord songé à inclure dans les Avantures d'Italie: «Cette pièce est un fragment que j'ay arraché de mes Avantures d'Italie, pour ce qu'il interrompait le cours de mon histoire. » Les Aventures burlesques..., op. cit., p. 325.

14. On pourrait encore citer ce passage où, sur le mode burlesque et équivoque qui lui est habituel, évoquant le difficile chemin qui le conduit à la Sainte Baume et dont Chapelle avait dit qu'il était tellement escarpé que le voyageur était tenté de se donner au diable pour arriver au bout, Dassoucy écrit : « bien que je ne sois pas plus devot que l'amy Chapelle, comme il ne couste pas plus de se donner à Dieu que de se donner au diable, je fis voir en me donnant à Dieu que j'avois le discernement meilleur que ce devot Pelerin. » (Av., p. 188-189).

15. Les Pensées..., op. cit., p. 332.

16. Victor Fournel, La Littérature indépendante et les écrivains oubliés, Paris, Didier, 1862, p. 147. Cité par Yves Giraud dans Charles Dassoucy, Les Amours d'Apollon et de Daphné, texte établi, présenté et annoté par Yves Giraud, Genève, Droz, Paris, Librairie Minard, 1969, p. 31.

17. Sur les problèmes posés par la lecture des textes « libertins », voir Jean-Pierre Cavaillé Dis/ simulations, Paris, H. Champion, 2000 et «Libertinage et dissimulation », Libertinage et philosophie au XVII siècle $n^{\circ} 5$ - Les libertins et le masque : simulation et représentation, Publication des Universités de Saint-Étienne, 2001, p. 57-82.

18. «Mystique. adj. Qui est mysterieux. Les Commentateurs de l'Ecriture outre le sens litteral y trouvent un sens mystique, allegorique et un sens moral. » Furetière, Dictionnaire universel.

19. Les Rimes redoublées de Monsieur Dassoucy, Paris, Nego, s.d. (1672 ou 1673). Sur les conditions de publication de cette deuxième édition et les problèmes d'autocensure qu'elle pose, voir Madeleine Alcover, op. cit.

20. Ibid., p. 3. Le Dictionnaire de l'Académie, 1694, précise: «On dit figurément, Mettre à la coupelle, Passer à la coupelle, pour dire, Mettre à une rigoureuse épreuve, Passer par un examen sévère. "

21. René Pintard, Le Libertinage érudit dans la première moitié du XVII siècle, Genève, Slatkine, nouvelle édition, 1983, p. 330.

22. J'emprunte l'expression à Pierre Brun dont la critique illustre bien cette circularité du jugement dans laquelle on enferme souvent Dassoucy et son œuvre : « Sa vie ! Elle est une longue burlesquerie elle-même et ses Aventures restent un bizarre compendium de tous les avatars que pouvait amener au XVII ${ }^{\mathrm{e}}$ siècle la vie de Bohème." Pierre Brun, Autour du XVII siècle, Genève, Slatkine, reprint, 1970, p. 53.

23. "Reste l'autoportrait anodin d'un aimable aventurier-gibier de potence qui n'a fait qu'appliquer la recette de ses contemporains. » estimait encore tout récemment Michel Jeanneret, Éros rebelle, Littérature et dissidence à l'âge classique, Paris, Seuil, 2003.

24. Philippe Lejeune, Le Pacte autobiographique, Paris, Seuil, 1975.

25. Jacques Berchtold, Les Prisons du roman (XVII ${ }^{e}$ XVIII ${ }^{e}$ siècle), Genève, Droz, 2000, p. 428.

26. Charles Dassoucy, Les Rimes redoubles de Monsieur Dassoucy, Paris, Nego, 1671, p. 20. 
27. Il faudrait discuter ici l'ouvrage de Joan Dejean, Libertines strategies, Freedom and the Novel in Seventeenth-Century France, Ohio State University Press, 1981, qui fait de la « persécution » une des clefs essentielles de son étude ("'Persecution' is a key term for this study») mais se refuse à distinguer entre la persécution réelle de l'écrivain et la persécution fictionnelle du personnage ("The libertine novelist and the libertine hero alike speak obsessively about their victimization. » Préface, p. XI), et traite de façon identique les œuvres de Sorel, de Cyrano, de Tristan et de Dassoucy.

28. René Démoris reprend avec bonheur cette disjonction établie par Roland Barthes et l'applique aux auteurs de récits à la première personne. Les "écrivants » sont les "grands seigneurs " auteurs de mémoires historiques : «Ceux que leur nom destinait à faire l'histoire deviennent des écrivants que qualifie leur rapport aux choses, au rebours de l'écrivain défini par son rapport au texte. »Op. cit., p. 7. La distinction vaut particulièrement pour Dassoucy qui tire de ses seuls titres dans la république des lettres la légitimité de son entreprise autobiographique.

29. «Nom du personnage = nom de l'auteur. Ce seul fait exclut la possibilité de la fiction. Même si le récit est, historiquement complètement faux, il sera de l'ordre du mensonge (qui est une catégorie autobiographique) et non de la fiction. » Op. cit., p. 30.

30. «Chapitre premier - Dassoucy, partant de Paris pour aller servir leurs A. R. de Savoye rencontre un Filou... » (Av., p. 33) ; «Chapitre II - Dassoucy après avoir tout perdu, passe luy mesme pour Filou auprés de celui qui l'a filouté... » (Av., p. 39) ; etc.

31. Un exemple parmi d'autres : «[...] je luy présentay tous mes ouvrages burlesques dans trois livres differens [...] - Quoy me dit-il, estes-vous l'Autheur de ces livres ? - Oüy, Monsieur, lui disje. - Vous estes donc Monsieur Dassoucy ?... (Av., p. 64).

32. Jean-Marie Schaeffer, Pourquoi la fiction ?, Paris, Seuil, 1999.

33. Sur les usages libertins de la fiction, voir Laurence Giavarini, «Le libertin et la fictionsorcière à l'âge classique : essai de mise au point ", dans Usages et théories de la fiction. XVI $I^{e}-X V I I I^{e}$ siècles, travaux du Clam (Paris VII), Presses Universitaires de Rennes, à paraître début 2004.

34. Philippe Lejeune, L'Autobiographie en France, France, Armand Colin, 1971, p. 43.

35. On n'a pas retrouvé le texte de Loret auquel Dassoucy consacre le chapitre VI des Avantures d'Italie sous le titre-résumé : « Comme Dassoucy arriva à Thurin où estant crû mort, d'abord il fut pris pour une ombre : il insulte contre Loret, et la tolérance des libelles» (p. 231). Yves Giraud signale une chanson anonyme composée après le supplice de Chausson et Fabry (1661) : "Si le bougre Dassouci / Eut été pris (bis) / Il aurait été rôti / Tout au travers des flammes / Comme ces deux infâmes / De Chausson et Fabri ». Yves Giraud, op. cit., p. 58.

36. J'emprunte l'expression à Jacques Prévot, op. cit., p. 1506.

37. «Le plus mauvais plaisant eut des approbateurs / Et jusqu'à Dassoucy, tout trouva des lecteurs. ", Boileau, Art poétique, Chant I, v. 89-90.

38. C'est assez dire que je ne partage pas le jugement de Jean Serroy qui réduit cet usage des codes du burlesque à une insuffisance d'invention créatrice : "Si d'Assoucy choisit d'assimiler son expérience vécue à un roman, et s'il donne à ce roman les caractères conventionnels de l'Histoire comique, c'est parce qu'il est incapable de concevoir un autre mode narratif. " Jean Serroy, Roman et réalité - Les histoires comiques au XVII ${ }^{e}$ siècle, Paris, Minard, 1981.

39. Roland Barthes, «La face baroque », Le Bruissement de la langue, Essais critiques IV, Paris, Le Seuil, coll. « Point Essais », 1984, p. 285.

40. Dominique Bertrand, éd., Poétiques du burlesque, Actes du Colloque international du Centre de Recherches sur les Littératures Modernes et Contemporaines de l'Université Blaise Pascal, (22-24 février 1996), Paris, Champion, 1998. 